\title{
A new estimator for vector velocity estimation [medical ultrasonics]
}

\author{
Jensen, Jørgen Arendt
}

Published in:

I E E E Transactions on Ultrasonics, Ferroelectrics and Frequency Control

Link to article, DOI:

10.1109/58.935705

Publication date:

2001

Document Version

Publisher's PDF, also known as Version of record

Link back to DTU Orbit

Citation (APA):

Jensen, J. A. (2001). A new estimator for vector velocity estimation [medical ultrasonics]. I E E E Transactions on Ultrasonics, Ferroelectrics and Frequency Control, 48(4), 886-894. https://doi.org/10.1109/58.935705

\section{General rights}

Copyright and moral rights for the publications made accessible in the public portal are retained by the authors and/or other copyright owners and it is a condition of accessing publications that users recognise and abide by the legal requirements associated with these rights.

- Users may download and print one copy of any publication from the public portal for the purpose of private study or research.

- You may not further distribute the material or use it for any profit-making activity or commercial gain

- You may freely distribute the URL identifying the publication in the public portal

If you believe that this document breaches copyright please contact us providing details, and we will remove access to the work immediately and investigate your claim. 


\title{
A New Estimator for Vector Velocity Estimation
}

\author{
Jørgen Arendt Jensen, Member, IEEE
}

\begin{abstract}
A new estimator for determining the twodimensional velocity vector using a pulsed ultrasound field is derived. The estimator uses a transversely modulated ultrasound field for probing the moving medium under investigation. A modified autocorrelation approach is used in the velocity estimation. The new estimator automatically compensates for the axial velocity when determining the transverse velocity. The estimation is optimized by using a lag different from one in the estimation process, and noise artifacts are reduced by averaging RF samples. Further, compensation for the axial velocity can be introduced, and the velocity estimation is done at a fixed depth in tissue to reduce the influence of a spatial velocity spread. Examples for different velocity vectors and field conditions are shown using both simple and more complex field simulations. A relative accuracy of $10.1 \%$ is obtained for the transverse velocity estimates for a parabolic velocity profile for flow transverse to the ultrasound beam and a SNR of $20 \mathrm{~dB}$ using 20 pulse-echo lines. The overall bias in the estimates was $-4.3 \%$.
\end{abstract}

\section{INTRODUCTION}

$\mathrm{M}$ EDICAL ultrasound is extensively used for studying blood flow dynamics in the human body by using color flow mapping. The technique displays a color image of the flow superimposed on an anatomic B-mode image. The velocity component along the ultrasound beam direction is measured, and a flow transverse to the beam is not displayed. This is shown in Fig. 1 in which flow in a carotid artery and jugular vein is displayed. The image was acquired with a convex array with radial beam lines, and the angle between flow direction and ultrasound beam therefore changes over the image. Notice the change of estimated flow direction around the dashed line in both vessels caused by the change of angle between the flow and the ultrasound beam. This angle dependence is one of the main limitations of current ultrasound color flow systems, because most vessels are parallel to the skin surface and it is therefore a problem to achieve a sufficiently small angle between the flow and the beam. Also, the flow is often not parallel to the vessel surface, and it is therefore difficult, if not impossible, to estimate the correct angle and compensate for it [1].

Manuscript received August 31, 1999; accepted January 16, 2001. This work was supported by grant 9700883 and 9700563 from the Danish Science Foundation, grant EF-632 from the Academy of Technical Sciences, and by B-K Medical A/S, Denmark.

The author is with Department Ørsted • DTU, Technical University of Denmark, DK-2800 Lyngby, Denmark (e-mail: jaj@oersted.dtu.dk).

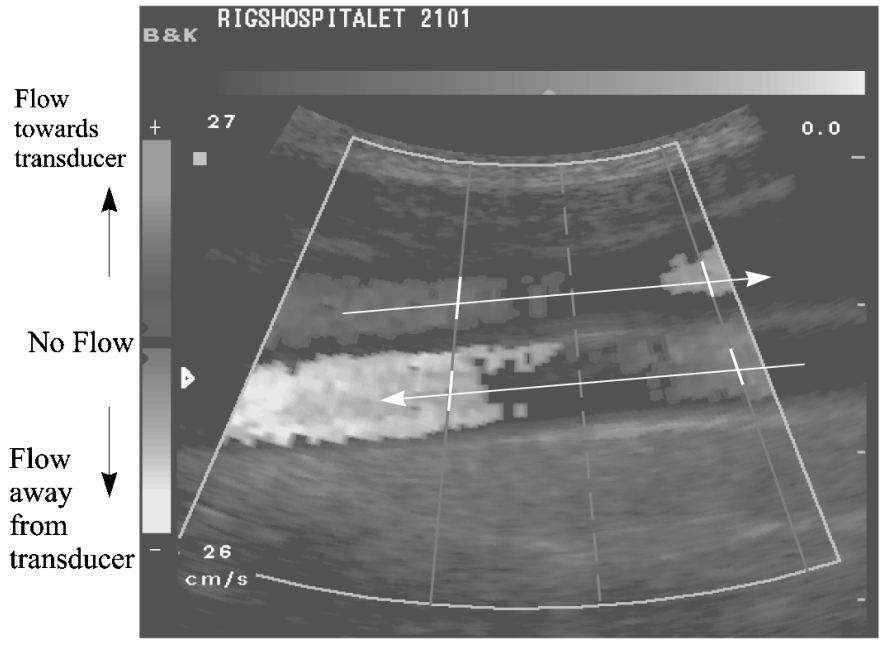

Fig. 1. Color flow image of the carotid artery and the jugular vein scanned with a convex array transducer. Notice the change of the angle between the ultrasound beam and the velocity vector around the dashed line.

Several authors have attempted to remedy this artifact. Fox [2] suggested using two beams to find the transverse component. The system works well for large transducers and investigations close to the transducer, but the variance of the transverse component estimate increases for situations with large depths and smaller transducers, such as those used in cardiac scanning through the ribs. Trahey et al. [3] have suggested using speckle tracking in which a small search region in one image is correlated or compared with a subsequent image. This approach has problems in terms of frame rate, because images are compared and the spatial resolution of the velocity estimates can be low. Newhouse et al. [4] developed a method in which the total bandwidth of the received signal is affected by the transverse velocity. It is, however, often difficult to find this bandwidth because of the inherent noise in the signal. Bonnefous [5] suggested using a number of beamformers working in parallel. The sample values at a given depth for the different beams are then used to form a signal transverse to the ultrasound beams. These transverse signals for different pulse emissions are then cross-correlated, and the transverse velocity is found from the shift in position of the maximum in the cross-correlation function. The method is, however, expensive because a number of parallel beamformers are used.

Jensen and Munk [6]-[8] have suggested introducing a transverse oscillation in the ultrasound field, whereby the transverse movement of the blood scatterers will give a 
modulation of the received signal. A similar approach has been suggested by Anderson [9], [10]. Here, the axial velocity must first be estimated and compensated for before the transverse velocity can be found, because the received signal is influenced by both transverse and axial velocity components.

In this paper, a new two-dimensional vector velocity estimator is explained. A brief description of traditional velocity estimation is given in Section II. The new estimator is derived in Section III along with various methods for further optimization. The performance of the estimator is evaluated in Section IV, both for simple monochromatic ultrasound fields and for full simulations of ultrasound fields and speckle patterns.

\section{Measurement of Blood Velocities}

In conventional color flow mapping systems, velocity estimation is performed by emitting a pulsed sinusoidal ultrasound field in one direction a number of times. The returned signal is then sampled at the depth of interest $d_{0}$. The sampled signal for a monochromatic, unit amplitude wave is given by [11]

$$
r(i)=\cos \left(2 \pi \frac{2 v_{z}}{c} f_{0} i T_{p r f}+\phi\right)
$$

where $c$ is the speed of sound, $v_{z}$ is the axial blood velocity component along the ultrasound beam direction, $f_{0}$ is the emitted center frequency, $i$ is the pulse number, $T_{p r f}$ is the time between pulse emissions, and $\phi$ is an arbitrary phase factor that depends on the depth. The frequency of the returned signal

$$
f_{p}=\frac{2 v_{z}}{c} f_{0}
$$

is, thus, proportional to the axial blood velocity component and can be found from either the mean frequency or the phase shift of the signal between pulse emissions. The velocity transverse to the ultrasound beam cannot be estimated from the sampled signal, and a signal influenced by the transverse velocity must be used. The underlying mechanism making it possible to perform axial velocity estimation is the oscillations in the emitted signal. Introducing a transverse oscillation in the ultrasound field makes the transverse velocity influence the received signal. The received signal can then be written as

$$
r_{t}(i)=\cos \left(2 \pi f_{p} i T_{p r f}+\phi\right) \cos \left(2 \pi \frac{v_{x}}{d_{x}} i T_{p r f}\right)
$$

where $v_{x}$ is the transverse velocity and $d_{x}$ is the lateral modulation period. The frequency caused by the transverse motion is

$$
f_{x}=\frac{v_{x}}{d_{x}}
$$

Such an approach has been suggested in [7], [8], [12].
Because velocities can be both positive and negative, a signal with a one-sided spectrum should be employed to probe the region of interest. This can be found by performing a Hilbert transform on the signals. For axial velocity estimation, the sampled signal is then

$$
r_{q}(i)=\exp \left(j 2 \pi \frac{2 v_{z}}{c} f_{0} i T_{p r f}+\phi\right)
$$

A spatial Hilbert transform can be employed for finding the transverse velocity. This can be approximated by simultaneously having two parallel probing beams separated by a distance $d_{x} / 4$ to yield the spatial quadrature field [8].

\section{Derivation OF THE Estimator}

The received and sampled spatial quadrature signal can be written as

$$
r_{s q}(i)=\cos \left(2 \pi f_{p} i T_{p r f}\right) \exp \left(j 2 \pi f_{x} i T_{p r f}\right)
$$

assuming that both the temporal and spatial fields are monochromatic and of unit amplitude and neglecting the phase term $\phi$. The received field is, thus, influenced by both the axial and the transverse velocity. The influence from the axial velocity on the transverse estimate has previously been compensated for by using tracked data, but any error in tracking, because of a poor axial velocity estimate, can negatively influence the transverse velocity estimate. The purpose of the new estimator is to reduce this effect and, thereby, decrease the variance of the estimates.

\section{A. Basic Estimator}

Axial velocity compensation by tracking can be avoided by employing the more advanced estimator developed in this section.

The temporal Hilbert transform of (6) is

$$
r_{s q h}(i)=\sin \left(2 \pi f_{p} i T_{p r f}\right) \exp \left(j 2 \pi f_{x} i T_{p r f}\right)
$$

Rewriting (6) and (7) using Euler's equations gives

$$
\begin{aligned}
r_{s q}(i)= & \frac{1}{2}\left(\exp \left(j 2 \pi i T_{p r f}\left(f_{x}+f_{p}\right)\right)\right. \\
& \left.+\exp \left(j 2 \pi i T_{p r f}\left(f_{x}-f_{p}\right)\right)\right) \\
r_{s q h}(i)= & \frac{1}{2 j}\left(\exp \left(j 2 \pi i T_{p r f}\left(f_{x}+f_{p}\right)\right)\right. \\
& \left.-\exp \left(j 2 \pi i T_{p r f}\left(f_{x}-f_{p}\right)\right)\right) .
\end{aligned}
$$


Two new signals are then formed from

$$
\begin{aligned}
r_{1}(i)= & r_{s q}(i)+j r_{s q h}(i) \\
= & \frac{1}{2}\left(\exp \left(j 2 \pi i T_{p r f}\left(f_{x}+f_{p}\right)\right)\right. \\
& \left.+\exp \left(j 2 \pi i T_{p r f}\left(f_{x}-f_{p}\right)\right)\right) \\
+ & j \frac{1}{2 j}\left(\exp \left(j 2 \pi i T_{p r f}\left(f_{x}+f_{p}\right)\right)\right. \\
& \left.-\exp \left(j 2 \pi i T_{p r f}\left(f_{x}-f_{p}\right)\right)\right) \\
= & \exp \left(j 2 \pi i T_{p r f}\left(f_{x}+f_{p}\right)\right) \\
r_{2}(i)= & r_{s q}(i)-j r_{s q h}(i) \\
= & \frac{1}{2}\left(\exp \left(j 2 \pi i T_{p r f}\left(f_{x}+f_{p}\right)\right)\right. \\
& \left.+\exp \left(j 2 \pi i T_{p r f}\left(f_{x}-f_{p}\right)\right)\right) \\
- & j \frac{1}{2 j}\left(\exp \left(j 2 \pi i T_{p r f}\left(f_{x}+f_{p}\right)\right)\right. \\
& \left.-\exp \left(j 2 \pi i T_{p r f}\left(f_{x}-f_{p}\right)\right)\right) \\
= & \exp \left(j 2 \pi i T_{p r f}\left(f_{x}-f_{p}\right)\right) .
\end{aligned}
$$

Finding the changes in phase as a function of sample number for the two signals gives

$$
\begin{aligned}
d \Theta_{1} & =2 \pi T_{p r f}\left(f_{x}+f_{p}\right) \\
d \Theta_{2} & =2 \pi T_{p r f}\left(f_{x}-f_{p}\right) .
\end{aligned}
$$

Adding the two phase changes gives

$$
d \Theta_{1}+d \Theta_{2}=2 \pi 2 T_{p r f} f_{x}=4 \pi T_{p r f} \frac{v_{x}}{d_{x}},
$$

and subtracting them gives

$$
d \Theta_{1}-d \Theta_{2}=2 \pi 2 T_{p r f} f_{p}=4 \pi T_{p r f} \frac{2 v_{z}}{c} f_{0} .
$$

The transverse velocity can, thus, be found directly from

$$
v_{x}=\frac{\left(d \Theta_{1}+d \Theta_{2}\right) d_{x}}{2 \pi 2 T_{p r f}},
$$

and the axial velocity can be found from

$$
v_{z}=\frac{\left(d \Theta_{1}-d \Theta_{2}\right) c}{2 \pi 4 T_{p r f} f_{0}} .
$$

The combination of signals makes it possible to automatically compensate for the axial and transverse movements. This is especially important for the transverse estimation because of the rapid variation in the phase for axial motion compared with transverse motion. An alternative to (15) is to find the axial velocity through a traditional estimator by forming a received beam with conventional beamforming without the transverse modulation. This can potentially yield a higher precision at the expense of an extra receive beamformer.

The determination of the phase changes for the complex signal can, e.g., be done using the standard autocorrelation estimator [11], [13]. Given a complex signal,

$$
r(i)=x(i)+j y(i) \text {, }
$$

the phase change is determined by

$$
d \hat{\Theta}=\arctan \left(\frac{\sum_{i=0}^{N-1} y(i) x(i-1)-y(i-1) x(i)}{\sum_{i=0}^{N-1} x(i) x(i-1)+y(i) y(i-1)}\right)
$$

Using the estimated complex autocorrelation of the signal

$$
\hat{R}(m)=\frac{1}{N-m} \sum_{i=0}^{N-m} r^{*}(i) r(i+m)
$$

this can also be stated as

$$
d \hat{\Theta}=\arctan \left(\frac{\Im\{R(1)\}}{\Re\{R(1)\}}\right)
$$

where $\Im\{R(1)\}$ denotes the imaginary part of the complex autocorrelation and $\Re\{R(1)\}$ is the real part both at a lag of 1 . This is equivalent to finding the mean frequency in the power density spectrum given by [11]:

$$
\bar{f}=\frac{\int_{-f_{p r f} / 2}^{f_{p r f} / 2} f P(f) d f}{\int_{-f_{p r f} / 2}^{f_{p r f} / 2} P(f) d f}
$$

where $R(m) \leftrightarrow P(f)$. Hereby, the axial velocity is determined by

$$
v_{z}=\frac{c}{2} \frac{\bar{f}}{f_{0}} .
$$

This estimator, thus, finds the mean velocity. The estimator is also unbiased for white noise added to the input signal $r(i)$ [11].

Finding the phase change by (17) entails finding the arc-tan of the argument, and the transverse velocity estimation through (14), thus, depends on two arc-tangents. This creates problems when the phase is greater than $\pi$. An improved calculation using the relationship

$$
\tan (A+B)=\frac{\tan (A)+\tan (B)}{1-\tan (A) \tan (B)}
$$

yields

$$
\begin{aligned}
& \tan \left(d \Theta_{1}+d \Theta_{2}\right)= \tan \left(\arctan \left(\frac{\Im\left\{R_{1}(1)\right\}}{\Re\left\{R_{1}(1)\right\}}\right)\right. \\
&\left.+\arctan \left(\frac{\Im\left\{R_{2}(1)\right\}}{\Re\left\{R_{2}(1)\right\}}\right)\right) \\
&= \frac{\frac{\left.\Im R_{1}(1)\right\}}{\Re\left\{R_{1}(1)\right\}}+\frac{\Im\left\{R_{2}(1)\right\}}{\Re\left\{R_{2}(1)\right\}}}{1-\frac{\left.\Im R_{1}(1)\right\}}{\Re\left\{R_{2}(1)\right\}}} \\
&= \frac{\Im\left\{R_{1}(1)\right\}}{\Re\left\{R_{2}(1)\right\}} \\
& \Re\left\{R_{1}(1)\right\} \Re\left\{R_{2}(1)\right\}-\Im\left\{R_{2}(1)\right\} \Re\left\{R_{1}(1)\right\} \\
&
\end{aligned}
$$




$$
v_{x}=\frac{d_{x}}{2 \pi 2 T_{p r f}} \arctan \left(\frac{\Im\left\{R_{1}(1)\right\} \Re\left\{R_{2}(1)\right\}+\Im\left\{R_{2}(1)\right\} \Re\left\{R_{1}(1)\right\}}{\Re\left\{R_{1}(1)\right\} \Re\left\{R_{2}(1)\right\}-\Im\left\{R_{1}(1)\right\} \Im\left\{R_{2}(1)\right\}}\right)
$$

and

$$
v_{z}=\frac{c}{2 \pi 4 T_{p r f} f_{0}} \arctan \left(\frac{\Im\left\{R_{1}(1)\right\} \Re\left\{R_{2}(1)\right\}-\Im\left\{R_{2}(1)\right\} \Re\left\{R_{1}(1)\right\}}{\Re\left\{R_{1}(1)\right\} \Re\left\{R_{2}(1)\right\}+\Im\left\{R_{1}(1)\right\} \Im\left\{R_{2}(1)\right\}}\right) .
$$

where $R_{1}(1)$ is the complex lag 1 autocorrelation value for $r_{1}(i)$ and $R_{2}(1)$ is the complex lag 1 autocorrelation value for $r_{2}(i)$. A similar expression can be derived for the axial velocity, and the estimators are as shown above in (24) and (25).

\section{B. Compensation for Different Wavelengths}

The lateral modulation period will, in general, be larger than the wavelength of the probing ultrasound pulse. For a given velocity, the change in phase for the transverse signal will, thus, generally be smaller than the change in phase for the axial signal. Optimizing the pulse repetition time for both measurements simultaneously is, therefore, not possible. However, a larger transverse phase change can be measured by using a lag $k>1$ in the autocorrelation estimator. The phase estimator,

$$
\begin{aligned}
d \hat{\Theta} & =\frac{1}{k} \arctan \left(\frac{\Im\{R(k)\}}{\Re\{R(k)\}}\right) \\
\hat{R}(k) & =\frac{1}{N-k} \sum_{i=0}^{N-k} r^{*}(i) r(i+k),
\end{aligned}
$$

can be directly used in (24) and (25) instead of (19).

Using the condition that the phase shift should be the same for $v_{x}=v_{z}$, the lag can be roughly determined by

$$
\begin{aligned}
& k \approx \frac{d_{x}}{\lambda} \\
& \lambda=\frac{c}{f_{0}} .
\end{aligned}
$$

Often this equation will give a large value for $k$, and the calculation of the autocorrelation in (26) will include too few values for a low variance estimate. A compromise can be attained by reducing $k$ to obtain both a larger phase shift and a sufficient number of data values for the calculation of $\hat{R}(k)$.

\section{Optimization in the Case of Noise and Attenuation}

The scattering of ultrasound from blood is weak, and the SNR is often poor after stationary echo canceling [11]. Therefore, a prime concern is to make the estimator robust in a noisy environment. This can be attained by averaging the autocorrelation estimate over the length of the interrogating pulse corresponding to performing a matched filtration. The length of the pulse in terms of RF samples is given by

$$
N_{p}=\frac{M}{f_{0}} f_{s}
$$

where $M$ is the number of periods in the pulse (typically 4 to 8 ) and $f_{s}$ is the sampling frequency. The autocorrelation estimate is then calculated by

$$
\begin{aligned}
\hat{R}(k)=\frac{1}{(N-k) N_{p}} \sum_{i=0}^{N-k} \sum_{n=-N_{p} / 2}^{N_{p} / 2-1} & r^{*}\left(i, n+N_{0}\right) r\left(i+k, n+N_{0}\right) .
\end{aligned}
$$

Here $r(i, n)$ denotes the received signal for the $i$ th line and its $n$th RF sample, $N_{0}$ is the sample number for the position of the velocity estimate, and the averaging is done symmetrically around this position to reduce the effects of velocity spread.

The center frequency $f_{0}$ of the ultrasound pulse will change as a function of depth because of the frequencydependent attenuation of the tissue. Therefore, the number of samples $N_{p}$ should also change. The actual mean center frequency can be determined by

$$
\bar{f}_{0}(z)=\frac{\int_{-f_{s} / 2}^{f_{s} / 2} f P_{r f}(f, z) d f}{\int_{-f_{s} / 2}^{f_{s} / 2} P_{r f}(f, z) d f}
$$

where $f_{s}$ is the sampling frequency, $z$ is the depth of interest, and $P_{r f}(f, z)$ is the spectrum of the received RF signal around the depth $z \cdot \bar{f}_{0}(z)$ can then be used in (28) to fit the filtration to the measurement situation as suggested by Loupas et al. [14].

\section{Using Axial Velocity Compensation}

The derivation of the estimators is done using monochromatic signals with a single velocity component, and the implicit compensation for the axial velocity is only valid for a single velocity. The estimate will be influenced by a velocity spread within the range gate, and making an axial velocity compensation can improve the estimates, 

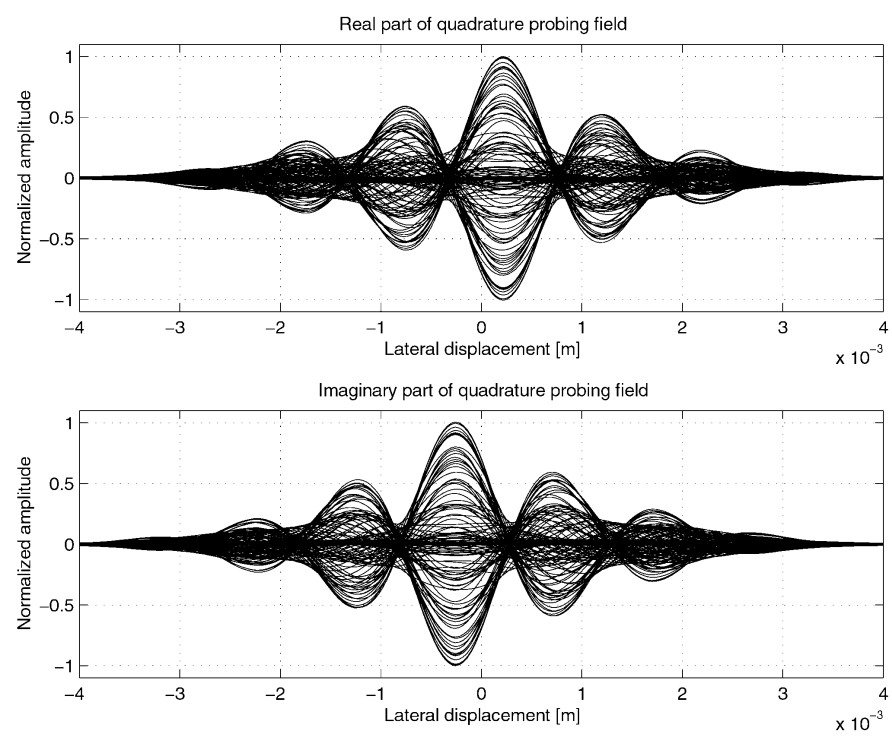

Fig. 2. In-phase (top) and quadrature (bottom) components of probing field as a function of lateral displacement. The lateral signal is shown for the full-time extent of the probing field.

when the signals contain such a spread in velocity and when a fairly broadband pulse is used. Introducing a compensation of a mean phase shift can be employed, because the developed estimator essentially subtracts a phase shift from the axial motion during the transverse estimation process. Whether this is the actual phase shift or a smaller phase shift is of no importance. The autocorrelation estimator finds the phase shift from one line to the next, and, therefore, a fixed phase shift or equivalent delay can be used. The autocorrelation estimator is then given by

$$
\begin{aligned}
& \hat{R}(k)=\frac{1}{(N-k) N_{p}} \sum_{i=0}^{N-k} \sum_{n=-N_{p} / 2}^{N_{p} / 2-1} r^{*}\left(i, n+N_{0}-n_{s} / 2\right) \\
& r\left(i+k, n+N_{0}+n_{s} / 2\right)
\end{aligned}
$$

where $n_{s}$ is the axial movement compensation delay given by

$$
n_{s}=\operatorname{round}\left(k \frac{2 v_{z}}{c} T_{p r f} f_{s}\right)
$$

rounded off to the nearest number of samples. This ensures that the estimator has the smallest phase shift from axial motion for which to compensate.

\section{E. Estimating the Lateral Modulation Period}

The use of an incorrect modulation period $d x$ in (24) will give rise to a biased estimate, because the transverse velocity estimate is directly proportional to the lateral modulation period.

The lateral modulation does not have so narrow a bandpass spectrum as the spectrum for the axial pulse. This can be seen from a plot of the lateral field in Fig. 2 and from the Fourier transform of the ultrasound field shown in Fig. 3. The autocorrelation method, however, estimates the mean

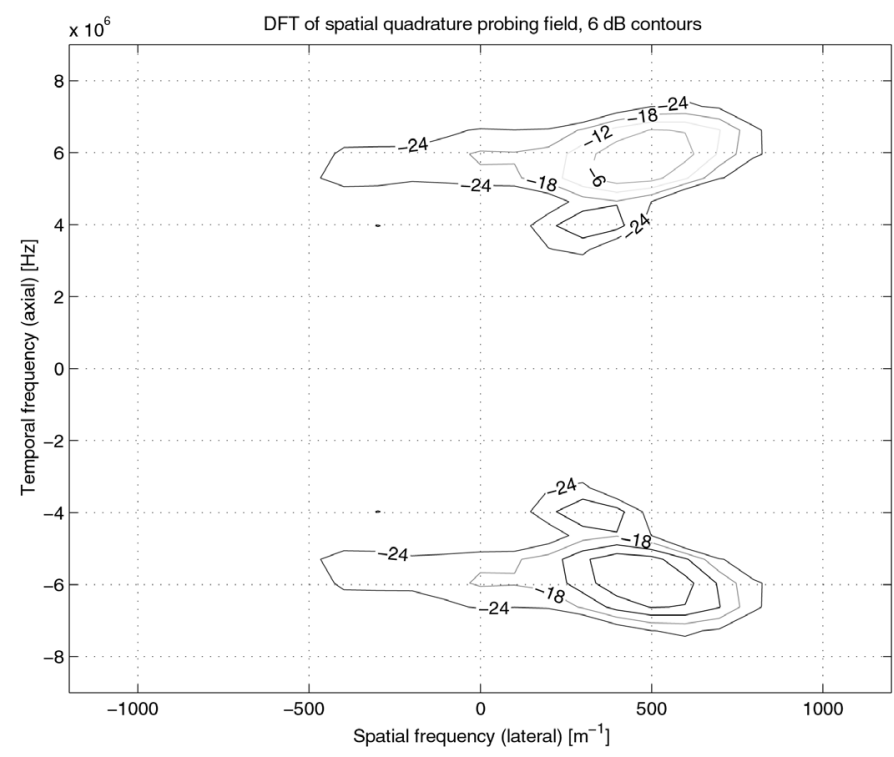

Fig. 3. Spatio-temporal Fourier transform of the complex probing field. A contour plot of the normalized amplitude spectrum is shown with $6 \mathrm{~dB}$ between contours.

frequency, and an estimate of the mean modulation period can, thus, be used to ensure unbiased estimates. This is obtained by first simulating or measuring both the inphase and quadrature field from a point scatterer moving in front of the transducer at the depth of interest. The spatio-temporal Fourier transform $H\left(f_{\text {time }}, f_{\text {space }}\right)$ of the complex probing field is then found as shown on Fig. 3. The real part of the transformed signal is the in-phase field, and the imaginary part is the quadrature component. The spectrum is approximately one sided because of the Hilbert transform relationship between the real and imaginary part of the signal. The mean spatial frequency can then be found from (33) (see next page) assuming that the scattering from the blood is homogeneous over the interrogated region and generates a white signal. Here, $f_{s x}$ is the lateral spatial sampling frequency. The mean lateral modulation period is then

$$
\bar{d}_{x}=\frac{1}{\bar{f}_{\text {space }}} .
$$

Fig. 3 and (33) also indicate how to optimize the measurement field. Ideally, a narrowband, single-sided spectrum should be used to yield a precise velocity estimate. A measure of the spectral spread is obtained by (35) (see next page). This can form the basis for the optimization of the spatial quadrature field, and minimizing $\sigma_{\bar{s}_{\text {space }}}^{2}$ will give the best result.

\section{Performance of Estimator}

This section presents a number of simulations used for revealing the performance of the new estimator under different operating conditions. This includes the use of purely monochromatic fields without noise to a full simulation using the simulation program Field II [15] of the diffraction effects of the ultrasound field and the speckle nature 


$$
\bar{f}_{\text {space }}=\frac{\int_{-f_{s} / 2}^{+f_{s} / 2} \int_{-f_{s x} / 2}^{+f_{s x} / 2} f_{\text {space }}\left|H\left(f_{\text {time }}, f_{\text {space }}\right)\right|^{2} d f_{\text {time }} d f_{\text {space }}}{\int_{-f_{s} / 2}^{+f_{s} / 2} \int_{-f_{s x} / 2}^{+f_{s x} / 2}\left|H\left(f_{\text {time }}, f_{\text {space }}\right)\right|^{2} d f_{\text {time }} d f_{\text {space }}}
$$

$$
\sigma_{\bar{f}_{\text {space }}}^{2}=\frac{\int_{-f_{s} / 2}^{+f_{s} / 2} \int_{-f_{s x} / 2}^{+f_{s x} / 2}\left(f_{\text {space }}-\bar{f}_{\text {space }}\right)^{2}\left|H\left(f_{\text {time }}, f_{\text {space }}\right)\right|^{2} d f_{\text {time }} d f_{\text {space }}}{\int_{-f_{s} / 2}^{+f_{s} / 2} \int_{-f_{s x} / 2}^{+f_{s x} / 2}\left|H\left(f_{\text {time }}, f_{\text {space }}\right)\right|^{2} d f_{\text {time }} d f_{\text {space }}}
$$

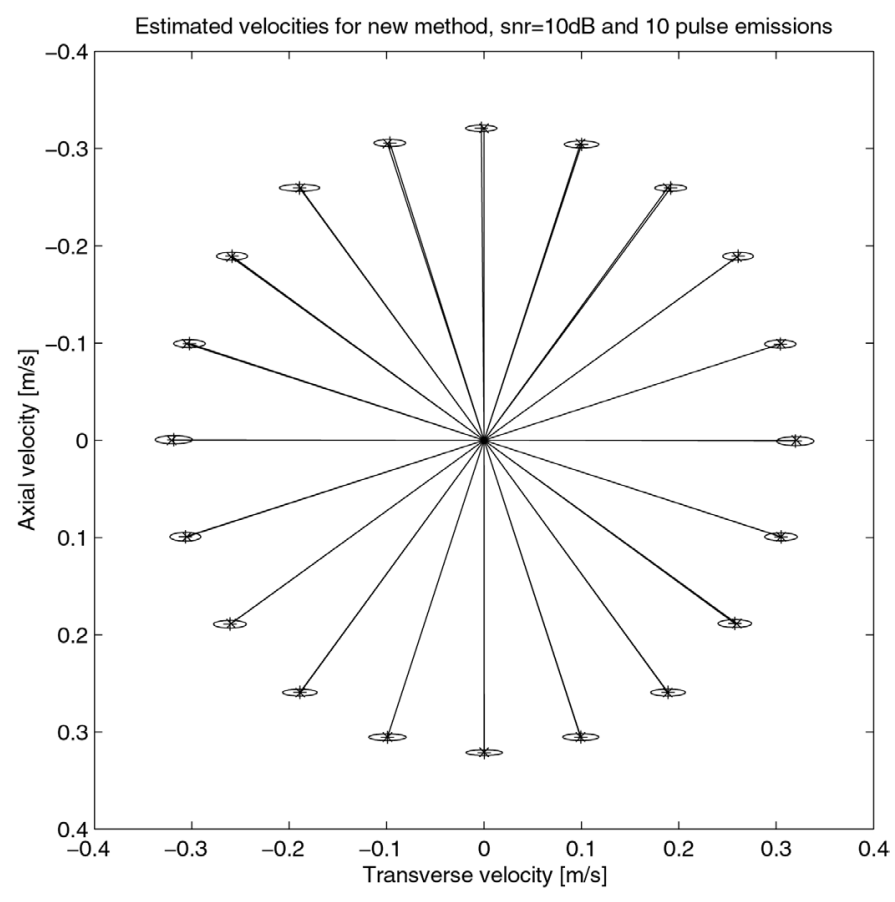

Fig. 4. Estimated velocity for a monochromatic field. The stars indicate the mean of the estimates, and the "+" show the correct velocity and direction. The ellipses show one standard deviation of the estimate.

of blood scattering for a parabolic flow profile with noise added.

The first example in Fig. 4 shows the performance of the new estimator for a purely monochromatic field, where the received signal is given by (6). The simulation parameters are given in Table I. Gaussian white noise was added to the signals. It is seen that the estimator correctly finds the velocity without error. No tracking is used in the estimator for this plot, but a similar performance is obtained with axial velocity compensation through tracking.

In the second example, a pulsed field generated by the Field II program is used [15], [16]. This field was convolved with a two-dimensional random, white Gaussian signal for generating a signal with speckle characteristics. The re-
TABLE I

Simulation Parameters for Monochromatic Simulation.

\begin{tabular}{lc}
\hline Parameter & Value \\
\hline$f_{s}$ & $50 \mathrm{MHz}$ \\
$f_{0}$ & $3 \mathrm{MHz}$ \\
$M$ & 4 \\
$d_{x}$ & $2 \mathrm{~mm}$ \\
$T_{p r f}$ & $100 \mu \mathrm{s}$ \\
$\mathrm{N}$ & 10 \\
$\mathrm{snr}$ & $10 \mathrm{~dB}$ \\
Lag compensation $k$ & 4 \\
\hline
\end{tabular}

ceived RF signals are then generated by selecting the appropriate data from this two-dimensional image according to the transverse and axial velocities. An integer number of samples is moved between pulse emissions in both directions. The simulation parameters are given in Table II. The results of the simulation are shown in Fig. 5 for a SNR of $20 \mathrm{~dB}$ and for 100 different estimates. The axial velocity was found using the normal autocorrelation estimator with RF averaging, and the new estimator was used for the transverse velocity. Axial velocity tracking was used in the new estimator. Increasing the noise to yield a SNR of $0 \mathrm{~dB}$ gives the results shown in Fig. 6 . Satisfactory results are still seen at this low SNR.

To avoid moving an integer number of samples in the generated speckle pattern, a full Field II simulation has been made using roughly 35000 point scatterers. The same parameters as before are used. Making a plug flow estimation for different angles gives the estimates shown in Fig. 7. No noise was added to these data, but similar results are obtained for $\mathrm{SNR}^{\mathrm{s}}$ above $10 \mathrm{~dB}$. There is a slight underestimation of the axial velocity for all angles. The reason for this is unknown. There is also a slight bias for the lateral estimates, but it is not consistent and can be due to statistical fluctuation in the data.

A full simulation with a parabolic flow profile using 36000 point scatterers was performed for a vessel with a radius of $5 \mathrm{~mm}$. The peak velocity was $0.5 \mathrm{~m} / \mathrm{s}$, and 


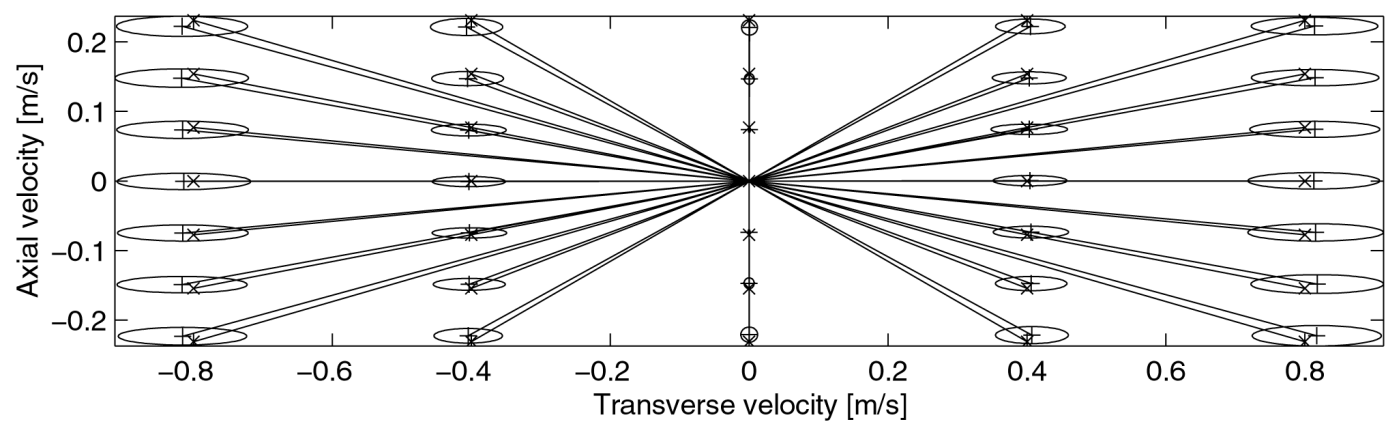

Fig. 5. Estimated velocity for speckle simulation. The stars indicate the mean of the estimates, and the "+" show the correct velocity and direction. The ellipses show one standard deviation of the estimate.

Estimates for method 5 (Field, axial), 20 emissions, snr =0dB, 100 estimates. 05-Jan-1999 21:57:33

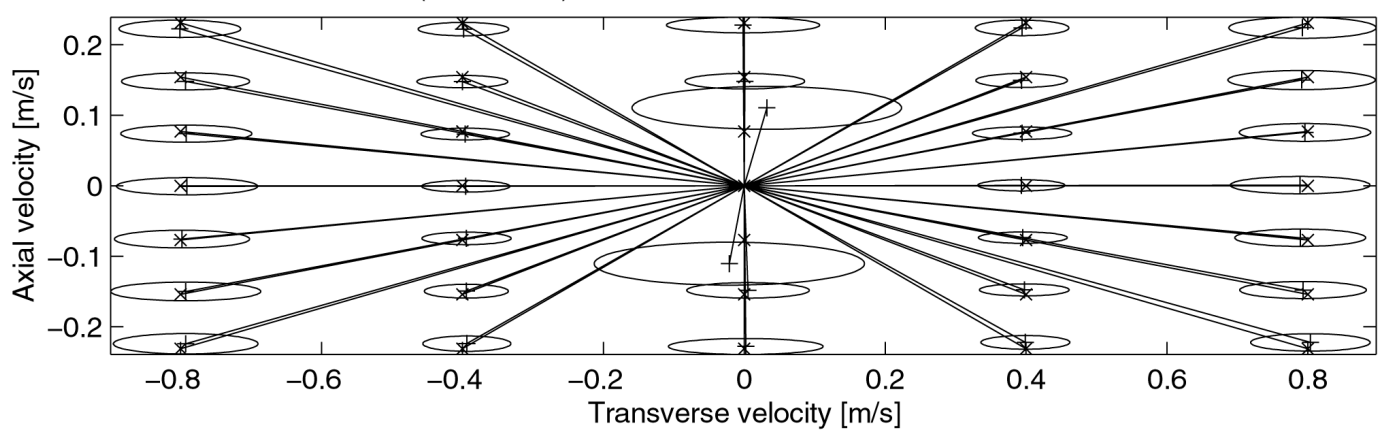

Fig. 6. Simulation result for a SNR ratio of $0 \mathrm{~dB}$. The stars indicate the mean of the estimates, and the "+" show the correct velocity and direction. The ellipses show one standard deviation of the estimate.

TABLE II

Parameters for Simulations USING FIELD II.

\begin{tabular}{lc}
\hline Parameter & Value \\
\hline$f_{s}$ & $100 \mathrm{MHz}$ \\
$f_{0}$ & $6 \mathrm{MHz}$ \\
$M$ & 4 \\
$\bar{d}_{x}$ & $2.26 \mathrm{~mm}$ \\
$T_{p r f}$ & $100 \mu \mathrm{s}$ \\
$N$ & 20 \\
snr & $20 \mathrm{~dB}$ \\
Lag compensation $k$ & 4 \\
Transducer elements & 128 \\
Transducer element width & $\lambda / 2$ \\
Transducer element height & $5 \mathrm{~mm}$ \\
Transducer element kerf & $\lambda / 10$ \\
Transmit focus & $70 \mathrm{~mm}$ \\
Transmit apodization & von Hann window \\
Receiver focus & fixed at $38.5 \mathrm{~mm}$ \\
Receiver apodization & double sinc \\
Center of vessel & $38.5 \mathrm{~mm}$ \\
\hline
\end{tabular}

the vessel was perpendicular to the ultrasound beam. A conventional color flow mapping (CFM) system would, in this situation, yield a velocity of 0 and, thus, would show that no velocity is present at this position in the image. The results from the new method are shown in Fig. 8. Gaussian noise was added in the simulation to obtain a SNR of $20 \mathrm{~dB}$; otherwise, the same parameters as before are used. The true velocity profile is shown as the dashed line. The standard deviation of the result is $0.050 \mathrm{~m} / \mathrm{s}$, and the mean deviation of the whole profile is -0.0067 $\mathrm{m} / \mathrm{s}$ compared with the true profile. The standard deviation relative to the maximum velocity is $10.1 \%$.

This simulation was repeated 50 times, and the average of all of the estimates is shown in Fig. 9. The standard deviation of all of the estimates averaged over the whole profile is $0.0303 \mathrm{~m} / \mathrm{s}$, corresponding to an accuracy of $6.07 \%$ relative to the peak velocity of $0.5 \mathrm{~m} / \mathrm{s}$. The overall mean deviation for the whole profile is $-0.0158 \mathrm{~m} / \mathrm{s}$ corresponding to a bias of $-3.16 \%$ relative to the peak velocity. The bias is most prominent after the peak value in the profile. The estimated lateral velocity is directly proportional to the lateral modulation period, which varies as a function of depth. A fixed set-up for the beamforming is used in the simulation, and the bias might be reduced by employing a dynamic beamforming, where the receive delays and apodization are adjusted dynamically, as the lateral modulation period is then kept constant as function of depth.

Fig. 10 shows the same simulation for a $60^{\circ}$ angle between the flow and the ultrasound beam. The standard deviation of all of the estimates averaged over the whole profile is now $0.13 \mathrm{~m} / \mathrm{s}$, corresponding to an accuracy of $25.4 \%$ relative to the peak velocity of $0.5 \mathrm{~m} / \mathrm{s}$. The overall mean deviation for the whole profile is $0.069 \mathrm{~m} / \mathrm{s}$, corresponding to a bias of $13.8 \%$ relative to the peak velocity. Estimating the angle between the beam and the flow 

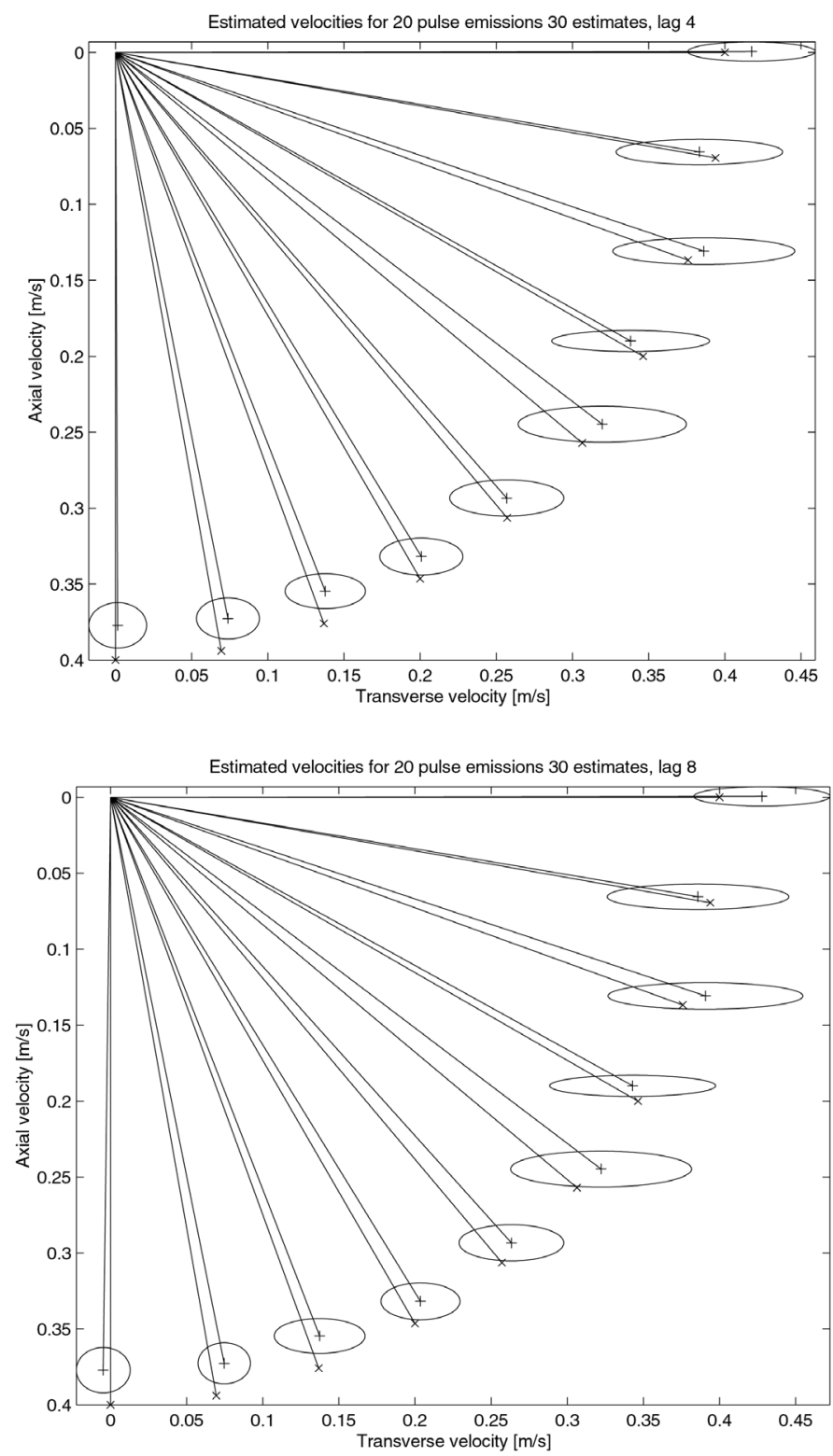

Fig. 7. Estimated velocities for different angles for plug flow. Two different lags (top, 4; bottom, 8) are used during the estimation process.

gives a mean angle of $67.0^{\circ}$ and a standard deviation of $17.6^{\circ}$ over the whole profile.

Thus, tilting the vessel significantly increases the standard deviation of the estimates, and this is due to the influence of the axial velocity, which is not completely eliminated by the estimator. Further work is still needed to make a more efficient compensation for the axial velocity that will decrease the variance for a spread of velocities.

\section{CONCLUSion}

A new estimator for finding the velocity transverse to the ultrasound beam has been developed. The estimator takes into account the influence from the axial velocity, both through a subtraction of phase shifts and through

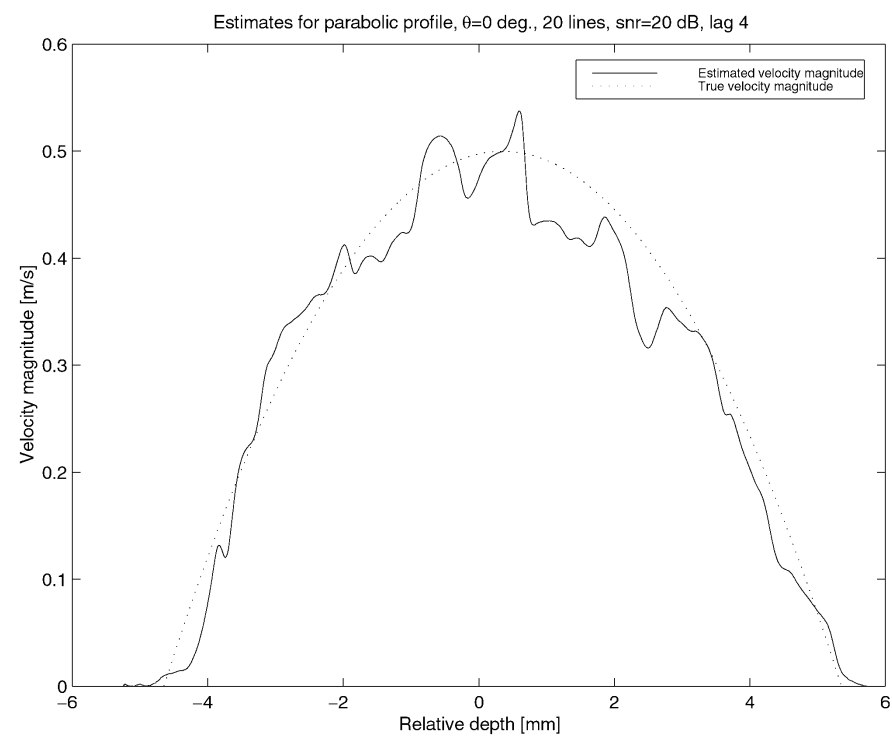

Fig. 8. Estimated parabolic velocity profile for flow perpendicular to the ultrasound beam.

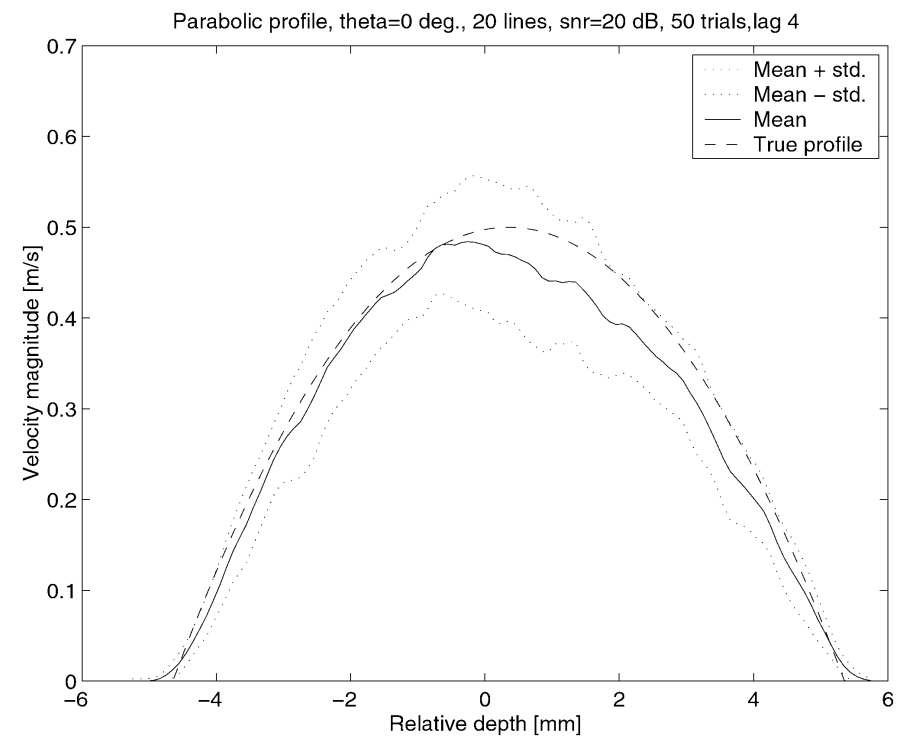

Fig. 9. Estimated parabolic velocity profile for flow perpendicular to the ultrasound beam. Mean of 50 estimates \pm one standard deviation.

tracking. The tracking is done between consecutive lines to minimize the axial range of tracking, thereby minimizing the effect of velocity dispersion. The estimator also partly compensates for the difference in modulation period of the axial and transverse modulation by incorporating a lag different from 1 as used in the traditional autocorrelation approach. The effect of noise is taken into account through averaging RF samples over the pulse length. Estimating the actual center frequency before averaging also takes into account the effect from attenuation. The new estimator is unbiased because the actual mean modulation frequency of the transverse field is estimated before being applied in the estimator. This can be done because the estimator finds the mean velocity in the transverse direction. Various simulation results have been shown both 


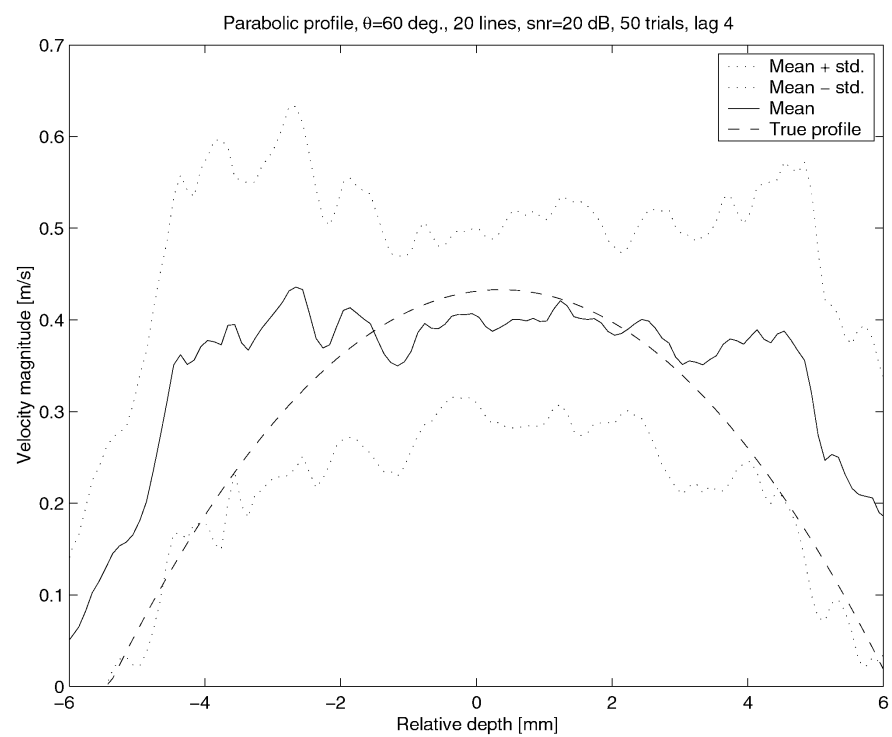

Fig. 10. Estimated parabolic velocity profile for flow with an angle of $60^{\circ}$ to the ultrasound beam. Mean of 50 estimates \pm one standard deviation.

for ideal fields and for very realistic fields simulated by the Field II program. Results for both a plug and a parabolic flow profile have been shown. The approach can find the velocity with a standard deviation of $10 \%$ relative to the maximum velocity, when the velocity is orthogonal to the ultrasound beam. For a tilted vessel, the axial velocity still influences the velocity estimation, and, for a $60^{\circ}$ angle, the standard deviation is $25.4 \%$.

\section{ACKNOWLEDGMENT}

The comments from the anonymous reviewers are greatfully acknowledged.

\section{REFERENCES}

[1] D. J. Phillips, K. W. Beach, J. Primozich, and D. E. Strandness, "Should results of ultrasound Doppler studies be reported in units of frequency or velocity?," Ultrasound Med. Biol., vol. 15, pp. 205-212, 1989.

[2] M. D. Fox, "Multiple crossed-beam ultrasound Doppler velocimetry," IEEE Trans. Sonics Ultrason., vol. SU-25, pp. 281286, 1978.

[3] G. E. Trahey, J. W. Allison, and O. T. von Ramm, "Angle independent ultrasonic detection of blood flow," IEEE Trans. Biomed. Eng., vol. BME-34, pp. 965-967, 1987.
[4] V. L. Newhouse, D. Censor, T. Vontz, J. A. Cisneros, and B. B. Goldberg, "Ultrasound Doppler probing of flows transverse with respect to beam axis," IEEE Trans. Biomed. Eng., vol. BME-34, pp. $779-788,1987$.

[5] O. Bonnefous, "Measurement of the complete (3D) velocity vector of blood flows," in Proc. IEEE Ultrason. Symp., pp. 795-799, 1988.

[6] J. A. Jensen, "Apparatus and method for determining movements and velocities of moving objects," International Patent PCT/DK97/00287, 1996.

[7] P. Munk, "Estimation of the 2-D flow vector in ultrasonic imaging: A new approach," M.S. Thesis, Dept. Inform. Technol., Technical University of Denmark, 1996.

[8] J. A. Jensen and P. Munk, "A new method for estimation of velocity vectors," IEEE Trans. Ultrason., Ferroelect., Freq. Contr., vol. 45, pp. 837-851, 1998.

[9] M. E. Anderson, "Spatial quadrature: A novel technique for multi-dimensional velocity estimation," in Proc. IEEE Ultrason. Symp., pp. 1233-1238, 1997.

[10] M. E. Anderson, "Multi-dimensional velocity estimation with ultrasound using spatial quadrature," IEEE Trans. Ultrason., Ferroelect., Freq. Contr., vol. 45, pp. 852-861, 1998.

[11] J. A. Jensen, Estimation of Blood Velocities Using Ultrasound: A Signal Processing Approach. New York: Cambridge University Press, 1996.

[12] P. Munk and J. A. Jensen, "Performance of a vector velocity estimator," in Proc. IEEE Ultrason. Symp., pp. 1489-1493, 1998.

[13] C. Kasai, K. Namekawa, A. Koyano, and R. Omoto, "Real-time two-dimensional blood flow imaging using an autocorrelation technique," IEEE Trans. Sonics Ultrason., vol. 32, pp. 458-463, 1985.

[14] T. Loupas, J. T. Powers, and R. W. Gill, "An axial velocity estimator for ultrasound blood flow imaging, based on a full evaluation of the Doppler equation by means of a two-dimensional autocorrelation approach," IEEE Trans. Ultrason., Ferroelect., Freq. Contr., vol. 42, pp. 672-688, 1995.

[15] J. A. Jensen, "Field: A program for simulating ultrasound systems," in Proc. 10th Nordic-Baltic Conf. Biomed. Imaging, vol. 4, Supplement 1, pt. 1, pp. 351-353, 1996.

[16] J. A. Jensen and N. B. Svendsen, "Calculation of pressure fields from arbitrarily shaped, apodized, and excited ultrasound transducers," IEEE Trans. Ultrason., Ferroelect., Freq. Contr., vol. 39, pp. 262-267, 1992.

Jørgen Arendt Jensen (M'93) earned his Master of Science in electrical engineering in 1985 and the Ph.D. degree in 1989, both from the Technical University of Denmark. He received the Dr. Techn. from the university in 1996. He has published a number of papers on signal processing and medical ultrasound and the book Estimation of Blood Velocities Using Ultrasound (Cambridge University Press, 1996). He has been a visiting scientist at Duke University, Stanford University, and the University of Illinois at Urbana-Champaign. He is currently full professor of biomedical signal processing at the Technical University of Denmark at the Department of Information Technology and head of Center for Fast Ultrasound Imaging. He has given courses on blood velocity estimation at both Duke University and University of Illinois and teaches biomedical signal processing and medical ultrasound imaging at the Technical University of Denmark. 